\title{
Politique
}

Politique

\section{Haute fonction publique et changement de gouvernement au Québec}

\author{
Le cas des sous-ministres en titre (1976-1989)
}

\section{Jacques Bourgault et Stéphane Dion}

Numéro 19, hiver 1991

URI : https://id.erudit.org/iderudit/040683ar

DOI : https://doi.org/10.7202/040683ar

Aller au sommaire du numéro

Éditeur(s)

Société québécoise de science politique

ISSN

0711-608X (imprimé)

1918-6584 (numérique)

Découvrir la revue

Citer cet article

Bourgault, J. \& Dion, S. (1991). Haute fonction publique et changement de gouvernement au Québec : le cas des sous-ministres en titre (1976-1989).

Politique, (19), 81-106. https://doi.org/10.7202/040683ar
Résumé de l'article

On peut distinguer deux types de haut fonctionnaire : premièrement, le grand commis haut fonctionnaire de carrière sans allégeance partisane et deuxièmement, l'outsider politique qui est recruté à l'extérieur de la fonction publique en fonction de son appartenance partisane. Le premier sert indifféremment tous les gouvernements, le second abandonne son poste avec le gouvernement qui l'a nommé. Lequel de ces deux modèles décrit le mieux le sous-ministre en titre du gouvernement du Québec? L’article répond à la question en examinant en quoi le profil des sous-ministres a été affecté par les changements de gouvernement de 1976 et 1985. Il apparaît que, tant sous les Péquistes que sous les Libéraux, les sous-ministres québécois ont été des grands commis " fragiles ». La plupart étaient des hauts fonctionnaires de carrière formellement apolitiques, mais très peu sont parvenus à demeurer en poste à la suite du changement de gouvernement.
Ce document est protégé par la loi sur le droit d'auteur. L'utilisation des services d’Érudit (y compris la reproduction) est assujettie à sa politique d'utilisation que vous pouvez consulter en ligne.

https://apropos.erudit.org/fr/usagers/politique-dutilisation/ 


\title{
Haute fonction publique et changement de gouvernement au Québec
}

Le cas des sous-ministres en titre (1976-1989)

\author{
Jacques Bourgault \\ Université du Québec à Montréal \\ Stéphane Dion* \\ Université de Montréal
}

On peut distinguer deux types de haut fonctionnaire : premièrement, le grand commis haut fonctionnaire de carrière sans allégeance partisane et deuxièmement, l'outsider politique qui est recruté à l'extérieur de la fonction publique en fonction de son appartenance partisane. Le premier sert indifféremment tous les gouvernements, le second abandonne son poste avec le gouvernement qui l'a nommé. Lequel de ces deux modèles décrit le mieux le sous-ministre en titre du gouvernement du Québec? L'article répond à la question en examinant en quoi le profil des sous-ministres a été affecté par les changements de gouvernement de 1976 et 1985 . II apparaît que, tant sous les Péquistes que sous les Libéraux, les sous-ministres québécois ont été des grands commis «fragiles». La plupart étaient des hauts fonctionnaires de carrière formellement apolitiques, mais très peu sont parvenus à demeurer en poste à la suite du changement de gouvernement.

On peut identifier deux modèles opposés de hauts fonctionnaires. À une extrémité du continuum de la carrière se retrouve le "grand commis de l'État" ou "grand fonctionnaire» (Gournay, $1978: 269-292)$. À l'autre extrémité, se situe l'outsider politique ou "l'étranger", appellation empruntée à Hugh Heclo (1977). La différence entre ces deux

* Les auteurs tiennent à remercier le CRSH, le Fonds FCAR et le Centre canadien de gestion pour leur contribution financière. Ils remercient également Catherine Beaudry, Manon Charron, Brigitte Ducharme, Michel Gagnon, Patrick Lutzy et Louise Thibault pour leur précieuse collaboration à différentes étapes de la recherche. 
modèles est basée sur trois critères. Tout d'abord, la permanence : le grand commis sert tous les gouvernements, quelle que soit leur couleur, tandis que l'outsider politique abandonne son poste en même temps que le gouvernement qui l'a nommé. Ensuite, l'identification politique : le grand commis n'a pas d'allégeance partisane connue alors que l'outsider politique est recruté en fonction de son appartenance partisane ou de ses affinités politiques ou personnelles avec les gouvernants de l'heure. Enfin, la provenance : le grand commis est recruté dans les services de l'État et y passe toute sa carrière, tandis que l'outsider, comme son nom l'indique, est recruté à l'extérieur de la fonction publique et est parachuté aux plus hauts postes de l'administration sans $y$ avoir fait carrière.

Comme ces trois critères ne sont pas forcément reliés entre eux, des catégories mixtes peuvent se ranger entre le type pur du grand commis et le type pur de l'outsider politique. Ce serait le cas, par exemple, d'un gestionnaire du secteur privé, qui n'aurait pas d'étiquette politique connue. Nommé directement haut fonctionnaire, il pourrait être qualifié d'outsider mais pas d'outsider politique.

L'objectif de ce texte est de vérifier si, dans un système politico-administratif donné, un changement de gouvernement peut modifier substantiellement le point d'équilibre entre le modèle du grand commis et celui de l'outsider politique. Le cas à l'étude sera celui des deux partis qui se sont échangé le pouvoir au Québec entre le 15 novembre 1976 et le 24 septembre 1989. Dans quelle mesure le Parti libéral a-t-il modifié le profil des sous-ministres en titre comparativement au Parti québécois? Nous répondrons à cette question après avoir précisé les particularités du cas québécois au cours des années 1976-1989, après avoir émis les hypothèses permettant de relier les changements de gouvernement avec les profils de hauts fonctionnaires et après avoir décrit notre banque de données. 


\section{Les particularités du cas québécois}

À priori, chaque modèle d'administrateur présente des avantages et des défauts (Dion, 1986 : 97-101). Le modèle du grand commis offre des gages d'expérience et de continuité, mais comporte les risques inhérents à une technocratie trop indépendante à l'égard du gouvernement en place. Inversement, celui de l'outsider politique présente l'avantage d'être relié sur les plans personnel et idéologique au pouvoir politique et à ses objectifs, mais il $n^{\prime}$ offre pas toutes les garanties d'expérience et d'autorité nécessaires dans la fonction publique. Chaque pays a arbitré à sa façon les avantages et les inconvénients des deux modèles. II est certain que, sur ce plan, le Québec et le Canada appartiennent à une tradition précise, celle de Whitehall.

Le modèle de l'outsider politique se rattache au système administratif américain, où I'administration de carrière est chapeautée par plusieurs échelons de political appointees, issus le plus souvent du secteur privé. Pour leur part, presque toutes les administrations européennes ont adopté un régime intermédiaire entre le grand commis et l'outsider politique, les ministères étant placés sous la direction administrative de fonctionnaires de carrière alignés sur le ou les partis au pouvoir. Ainsi, la proportion des hauts fonctionnaires officiellement affiliés à un parti politique a été estimée à $58 \%$ en Norvège (Lœgreid et Olsen, $1984: 229$ ), à $67 \%$ en Suisse (Urio et al., $1989: 62$ ), à $74 \%$ en Belgique et en Hollande (Dierickx, 1990 : 10; Elderweld et al., $1975: 135$ ), à $80 \%$ en RFA (Derlien, $1988: 73$ ) et à $100 \%$ en Suède (Linde et Ehn, $1990: 18)$.

Le modèle du grand commis est particulièrement prisé dans les pays de tradition britannique, où l'on trouve le plus souvent, à la tête des administrations, des fonctionnaires de grande expérience et sans allégeance partisane connue (Boreham, Cass et McCallum, 1979; Theakston et Fry, 1989). Ainsi, au gouvernement fédéral canadien (Bourgault et Dion, 1990), les 115 sous-ministres en titre, nommés entre 1967 et 1988, avaient, au moment de leur nomination, accumulé en moyenne dix-sept années de carrière dans la fonction publique 
fédérale, alors que seulement huit d'entre eux avaient exercé auparavant quelque activité politique officielle (candidature à une élection fédérale ou provinciale, participation à un cabinet ministériel, etc.). Bien sûr, les premiers ministres ont pu favoriser les candidats qui leur étaient sympathiques, mais ils ont recruté peu de partisans avoués et ont puisé principalement dans le réservoir des fonctionnaires de carrière.

Tout comme le gouvernement fédéral, celui du Québec s'est doté d'une haute administration formellement apolitique. Les nominations à caractère politique évident ont toujours été peu nombreuses, et leur proportion a été à la baisse entre 1939 et 1976 (Bourgault, 1986 : 360-370). Parmi les sousministres en titre et les sous-ministres adjoints et associés en poste entre 1960 et 1966, $32 \%$ avaient mené des activités politiques avant leur nomination; cette proportion tombe à $28 \%$ pour la période $1966-1970$ et à $9 \%$ pour la période 1970-1976.

Le gouvernement du Parti québécois a-t-il respecté les principes $d$ 'apolitisme formel et d'appartenance à la fonction publique dans le choix de ses hauts fonctionnaires? Louis Bernard, secrétaire général à l'époque, assure que l'appartenance à l'administration de carrière a été préservée. Selon lui (Bernard, 1987 : 101), $80 \%$ des postes d'administrateurs d'État (sous-ministres et sous-ministres adjoints et associés) ont été recrutés dans la fonction publique. Le recrutement extérieur a comblé un peu plus de $10 \%$ des postes, et la filière politique un peu moins de $10 \%$.

Le Parti libéral est retourné au pouvoir en décembre 1985 avec un discours très favorable au style de gestion du secteur privé et en annonçant des «mesures rigoureuses [...] pour endiguer la tendance naturelle de toute bureaucratie à vouloir bâtir des empires" (Parti libéral du Québec, 1985 : 18). Quelques mois après l'élection fut publié le Rapport Gobeil qui recommandait la réduction du nombre de hauts fonctionnaires dans les ministères (Rapport Gobeil 1986 : 40-41). Par la suite, de plus en plus de hauts fonctionnaires ont été embauchés par contrat selon des normes inspirées de la pratique du secteur privé (Bourgault et Dion, 1989). Les médias se sont demandé si cette procédure d'embauche n'ouvrait pas la voie 
à une plus grande politisation (Lesage, 1986). Le Parti québécois, lui, a prétendu que non seulement le gouvernement libéral confiait les postes de direction des conseils consultatifs, des régies, des offices et des sociétés d'État à des amis du régime, mais qu'il étendait la politisation jusqu'au centre névralgique de l'administration en dispensant les postes de sous-ministres en titre et de sous-ministres adjoints et associés à des partisans libéraux reconnus. La presse a repris cette critique avec, à la une, des titres inquiétants : "Nominations dans la fonction publique : Bourassa pratique le favoritisme sans se gêner" (Le Devoir, 23-01-88); "La haute fonction publique serait farcie de libéraux", (Le Devoir, 8-0489); "Avalanche de sous-ministres» (Le Soleil, 11-07-89). II semblerait donc, $d$ 'après ces informations, que le Parti libéral aurait singulièrement modifié le profil des sous-ministres, le rapprochant du modèle de l'outsider politique.

Au cours de notre étude, nous avons conduit une série de dix-sept entretiens semi-directifs afin de recueillir les points de vue de personnes ayant assisté depuis les premières loges à la transition politique de décembre 1985. Parmi ces observateurs rencontrés individuellement, on trouve six anciens chefs de cabinet en poste sous le Parti québécois, huit chefs de cabinet libéraux, un secrétaire général en poste sous le gouvernement du Parti québécois, un secrétaire général en poste sous le gouvernement du Parti libéral et un ancien ministre péquiste. Tous soulignent la façon professionnelle dont s'est opérée la transition, grâce surtout à l'étroite collaboration entre Louis Bernard et son successeur, Roch Bolduc. Cependant, nos interlocuteurs libéraux ont mentionné trois facteurs ayant incité le nouveau gouvernement à vouloir modifier en profondeur la haute fonction publique en place :

- Le sentiment que les hauts fonctionnaires laissés en place par le Parti québécois étaient des concepteurs de grandes politiques coûteuses plutôt que des gestionnaires rigoureux préoccupés par le déficit;

- Le sentiment que plusieurs hauts fonctionnaires étaient des péquistes inavoués; 
- Le complexe de supériorité que plusieurs libéraux éprouvent envers tout ce qui est identifié au style de gestion du Parti québécois.

Nos interlocuteurs libéraux ont nié que l'opération ait donné lieu au favoritisme partisan. Certains ont ajouté qu'elle a été conduite avec une grande prudence, afin de conserver la pleine collaboration de l'appareil administratif. "On ne voulait pas se mettre l'appareil à dos comme l'ont fait les conservateurs sur la scène fédérale; on jugeait plutôt chaque cas à son mérite de façon à conserver la collaboration des bons sous-ministres" (chef de cabinet libéral). Cette prudence a été jugée nécessaire dès qu'il est apparu que les sousministres allaient conserver toute leur puissance : "Après les premiers mois de transition, le pouvoir des hauts fonctionnaires est redevenu très important. À deux ou trois exceptions près, je ne transige pas avec les cabinets de ministre, mais plutôt soit avec les ministres, soit avec les sous-ministres» (chef de cabinet libéral).

Nos interlocuteurs péquistes ont eux aussi émis un point de vue assez homogène. Ils affirment que peu de sousministres laissés en place par le gouvernement du Parti québécois avaient une allégeance partisane ouverte, mais que beaucoup avaient une préférence connue pour la formation souverainiste, ou du moins étaient perçus comme tels, d'où leur remplacement rapide par des administrateurs plus proches des réseaux et des orientations libérales : "Dans les deux ministères où j'ai travaillé, les sous-ministres étaient pour nous, on le savait, et ils ont rapidement sauté après l'arrivée des libéraux" (chef de cabinet péquiste). Quelques-uns pensent que Robert Bourassa est allé plus loin dans la partisanerie et qu'il a étendu le patronage partisan depuis le rang de sous-ministre adjoint jusqu'à celui de sous-ministre en titre.

En somme, le cas québécois présenterait deux caractéristiques. La haute fonction publique au Québec se rapprocherait surtout du modèle du grand commis, avec une faible politisation explicite et un recrutement opéré le plus souvent à même I'effectif de la fonction publique. Mais le gouvernement libéral 
en place depuis décembre 1985 aurait procédé, semble-t-il, à des changements importants allant dans le sens du modèle de l'outsider politique. II s'agit donc maintenant de vérifier l'existence de ces changements et d'en mesurer la portée.

\section{Hypothèses et données}

À priori, viennent à l'esprit deux hypothèses non exclusives, selon lesquelles les changements de gouvernements auraient un effet sur le profil des hauts fonctionnaires. La première est celle d'un effet de parti : il peut $y$ avoir des divergences entre les partis qui se succèdent au pouvoir; en raison de leurs idéologies particulières ou de leurs réseaux d'influence, certaines formations politiques peuvent préférer le modèle du grand commis, et d'autres le modèle de l'outsider politique. La deuxième hypothèse est celle d'un effet de transition: les gouvernements fraîchement élus peuvent privilégier les outsiders politiques, par méfiance envers le personnel et les façons de faire de la haute administration laissée en place par l'ancien gouvernement. À leur arrivée au pouvoir, ils parachutent leurs propres outsiders politiques à la tête des administrations, quitte à renouer avec les fonctionnaires de carrière une fois leur apprentissage du pouvoir accompli.

II convient donc de tester la valeur explicative de ces deux hypothèses : l'effet de parti et l'effet de transition. La première prévoit un mouvement de balancier au gré des changements de parti au pouvoir, et la seconde un engouement exceptionnel mais passager pour le modèle de l'outsider politique. Mais il est aussi possible que le point d'équilibre entre le modèle du grand commis et celui de l'outsider politique évolue selon de tout autres facteurs, tels le marché de l'emploi ou les modes managériales, n'ayant rien à voir avec les changements de gouvernements.

Le cas du Québec des années 1976-1989 se prête bien à la démonstration. On peut délimiter trois périodes bien tranchées pendant lesquelles les premiers ministres en fonction ont eu tout le loisir de modifier à leur convenance le profil des hauts fonctionnaires : 1 ) le premier mandat du Parti 
québécois (16 novembre 1976 au 12 avril 1981), 2) le second mandat du Parti québécois (14 avril 1981 au premier décembre 1985) et 3 ) le premier mandat du Parti libéral (2 décembre 1985 au 24 septembre 1989). Ces périodes sont d'autant plus faciles à comparer qu'elles ont été à peu près de même durée : quatre ans et cinq mois pour le premier mandat du Parti québécois; quatre ans et sept mois pour le second mandat du Parti québécois; et trois ans et onze mois pour le mandat libéral.

Nous avons reconstitué les curriculum vitae des 102 sousministres en titre qui se sont succédé à la tête des ministères et des organismes centraux (Conseil exécutif et Conseil du trésor) durant ces trois périodes. Seuls les sous-ministres en titre font partie de notre étude, ce qui exclut les secrétaires généraux associés du Conseil exécutif, les sous-ministres adjoints et associés, ainsi que les intérimaires qui ont occupé leur poste pendant moins de six mois et qui par la suite $n^{\prime}$ ont pas été nommés sous-ministres en titre. L'étude porte donc sur les fonctionnaires au sommet de la hiérarchie à qui est confiée la première responsabilité administrative au sein d'un ministère ou d'un organisme central.

$C^{\prime}$ est à partir de diverses sources ${ }^{1}$ que nous avons recueilli les renseignements concernant le passé politique et professionnel des 102 sous-ministres en titre. En ce qui touche le passé politique, seules les activités officielles peuvent être repérées sur la base des informations disponibles : candidature à une élection, élection, poste de responsabilité dans un parti, participation à un cabinet politique. Une distinction doit être faite entre ces implications partisanes ouvertes et les fonctions ou allégeances politiques plus implicites, qui échappent à notre grille.

1. Nos données proviennent des sources suivantes : les communiqués hebdomadaires du bureau du Premier ministre. Bourgault, Le Répertoire des employés supérieurs (hors cadre) des ministères du gouvernement du Québec 1867-1983, 1984. Bourgault, Les administrateurs d'État du gouvernement du Québec 1867-1985, thèse de doctorat d'État, Paris, 1986. Gazette officielle du Québec, partie II, Lois et règlements, Éditeur officiel du Québec. Répertoire 1987 des fichiers des ministères et organismes du gouvernement du Québec, Commission d'accès à l'information, 1988. 
Appliquées au cas québécois, nos hypothèses s'énoncent de la façon suivante. Si, tant sur le plan de la permanence, de l'identification partisane que de la provenance des hauts fonctionnaires, le premier et le second mandat du Parti québécois se ressemblent et contrastent fortement avec le mandat libéral, nous conclurons que l'hypothèse 1 (l'effet de parti) est la plus plausible. Par contre, des résultats mettant en évidence une proportion d'outsiders politiques nettement plus élevée sous les premiers mandats du Parti québécois et du Parti libéral que sous le second mandat du Parti québécois iraient dans le sens de l'hypothèse 2 (I'effet de transition). Enfin, une évolution progressive devra plutôt être attribuée à une conjoncture indépendante des transitions de gouvernements.

Mais, avant de livrer nos résultats, une précision méthodologique s'impose. Les sous-ministres associés ont tous été exclus de l'étude bien que la loi donne à certains d'entre eux le statut de sous-ministre : c'est le cas, par exemple, au ministère de l'Éducation et pour certains secrétaires généraux associés du Conseil exécutif. Mais il n'a pas été possible de déterminer rigoureusement lesquels des secrétaires généraux associés agissent vraiment comme sous-ministres. En raison de ces difficultés d'analyse, aucun des sous-ministres associés ne fait partie de notre étude même si, du point de vue juridique ou fonctionnel, nous reconnaissons que certains cas auraient pu y être inclus.

Ce choix n'est pas sans conséquences. Comme l'un de nous l'a démontré dans une étude antérieure (Bourgault, 1986), la proportion de nominations partisanes tend à être d'autant plus élevée qu'on descend dans la hiérarchie des nominations discrétionnaires. C'est à l'échelon du sousministre que la tradition du grand commis est la mieux ancrée; c'est donc à cet échelon que toute évolution vers le modèle de l'outsider politique revêt le plus d'importance. 


\section{La permanence au rang occupé}

Le modèle du grand commis suppose qu'un gouvernement fraîchement élu garde à son service les hauts fonctionnaires laissés en place par le gouvernement précédent, afin de profiter de leur expérience et d'assurer une certaine continuité dans le traitement des affaires publiques. Le modele de l'outsider politique, au contraire, qui mise davantage sur la cohésion des élites dirigeantes et leur relève périodique, préconise le remplacement rapide des titulaires en poste par des partisans du nouveau pouvoir. Selon la loi, au Québec, les sous-ministres jouissent de la sécurité d'emploi, mais il est possible pour le gouvernement soit de leur suggérer de partir, soit de les muter à des postes de moindre responsabilité ou, encore, de les rétrograder.

Le tableau 1 permet de vérifier ce qui s'est passé sur ce plan entre 1976 et 1989 . II retrace le rythme des départs des sous-ministres qui étaient en fonction au début de chacun des trois mandats considérés. Un «départ» se produit lorsqu'un sous-ministre en titre cesse d'être à la tête de l'administration d'un ministère ou d'un organisme central, même quand il reste un employé du gouvernement du Québec en occupant une autre fonction. II est à noter que, dans ce tableau comme dans les suivants, les résultats sont livrés en pourcentages et en chiffres bruts. Les pourcentages doivent toutefois être utilisés avec précaution en raison du faible nombre de cas étudiés, mais ils n'en demeurent pas moins utiles pour comparer les mandats avec le maximum d'exactitude.

Du tableau 1 toujours, il ressort que le modèle du grand commis ne correspond guère à la réalité québécoise quant au critère de la permanence. Les péquistes, de 1976 à 1981, aussi bien que les libéraux, de 1985 à 1989, ont procédé au remplacement presque complet des sous-ministres laissés en place par le parti rival. Peu de sous-ministres ont franchi avec succès l'épreuve de l'alternance politique. La palme du "survivant" va à Robert Normand, le seul que le Parti québécois ait trouvé en fonction en 1976 et qu'il ait conservé sans interruption - d'ailleurs à des postes importants : Justice, 
Affaires intergouvernementales, Finances - jusqu'au retour des libéraux.

Tableau 1 Rythme des départs des sous-ministres en fonction au début du mandat

Mandat

Départs

Péquiste 1 Péquiste 2 Libéral

Lors des 6 premiers mois

3

$(14 \%)$

8 (36\%)

3 $(14 \%)$

5 $(23 \%)$

3 (14\%)

22 (101\%)

$\%)$

2

(9\%)

6

Du $7^{\bullet}$ au $12^{\bullet}$ mois

Du $13^{\circ}$ au $24^{\circ}$ mois

1

2

(5\%)

(8\%)

Du $25^{\circ}$ à la fin du mandat

Encore en fonction

à la fin du mandat

Total*

- Les pourcentages ayant été arrondis, leur total peut ne pas égaler $100 \%$. 
Des deux hypothèses à l'étude l'effet de transition est celle qui prédisait exactement ce que l'on trouve au tableau 1. Tandis que les nouveaux gouvernements de 1976 et de 1985 ont renouvelé leur personnel de sous-ministres, le gouvernement péquiste rélu en 1981 a conservé jusqu'à la fin la moitié des sous-ministres qu'il avait lui-même laissés en poste au terme de son premier mandat. II en est résulté une plus grande stabilité dans la composition de la haute fonction publique entre avril 1981 et décembre 1985 que lors des deux autres périodes considérées. Selon nos calculs, les ministères ont changé de sous-ministre exactement au même rythme lors du premier mandat péquiste et lors du mandat libéral, soit tous les 19 mois en moyenne, comparativement à tous les 26 mois lors du deuxième mandat du Parti québécois.

Quant à l'hypothèse d'une différenciation entre partis, elle est invalidée. Certes, le remaniement libéral a été plus rapide que le remaniement péquiste lors du premier mandat $125 \%$ de départs après six mois comparativement à $14 \%$ ). Sans doute l'équipe libérale de 1985 a-t-elle agi plus rapidement parce qu'elle avait une meilleure connaissance des personnes et des réseaux de la fonction publique (le Parti québécois de 1976 $n^{\prime}$ avait pas son Roch Bolduc). Mais le Parti québécois s'est rattrapé au cours des six mois suivants et, en fin de course, le phénomène a atteint la même intensité en 1976 qu'en 1985 : le changement de parti au pouvoir a donné lieu à un chambardement de l'élite administrative.

II ne semble pas que les sous-ministres en poste à l'arrivée au pouvoir du Parti québécois en 1976, ou à celle du Parti libéral en 1985, aient eu une claire identification politique au gouvernement sortant (ce qui aurait pu justifier leur éviction par le nouveau). D'après nos données, sur les 22 sous-ministres en fonction le 15 novembre 1976, 2 seulement avaient eu par le passé une activité politique officielle, soit comme employé du cabinet d'un ministre libéral (ils quitteront leurs fonctions respectivement dix et vingt mois après l'élection du Parti québécois). De même, sur les 24 sous-ministres en place à la prise du pouvoir par les libéraux en décembre 1985, seul le secrétaire général Louis Bernard avait officiellement un passé péquiste, ayant dirigé dans les années 70 les cabinets 
du chef de l'opposition et du Premier ministre. II a quitté son poste rapidement (le 22 décembre 1985), mais non sans que le Premier ministre Bourassa ait tenté de le retenir plus longuement, ce qui aurait facilité la transition (d'après les entrevues que nous avons faites). II est probable que plusieurs sousministres en place à l'arrivée des péquistes en 1976 et des libéraux en 1985 avaient une identification politique plus feutrée, plus implicite, avec l'ancien gouvernement, mais ils ne s'étaient pas écartés formellement du principe d'apolitisme qui caractérise le modèle du grand commis.

On ne peut pas dire non plus que ces sous-ministres en fonction avaient atteint l'âge de la retraite, ce qui, là encore, aurait pu expliquer le taux élevé de départs. Ceux qui ont quitté avant la fin de leur mandat avaient en moyenne, au moment de leur départ, 46 et 48 ans lors des premier et second mandats péquistes et 50 ans lors du mandat libéral. Seulement quatre d'entre eux avaient dépassé 55 ans, et trois sont partis sous le mandat libéral. La grande majorité avait encore de bonnes années de service à offrir.

Que sont devenus tous ces sous-ministres? Alors que le modèle du grand commis prévoit une réaffectation à d'autres postes de prestige, celui de l'outsider politique implique leur rétrogradation ou, tout simplement, leur départ. Le tableau 2 montre des résultats moins défavorables pour le modèle du grand commis que le tableau 1. En effet, les mobilités horizontales ont été nettement plus nombreuses que les rétrogradations. Le fait est que plusieurs sous-ministres ont été mutés à des postes de haute responsabilité, tels que président de SIDBEC, président de REXFOR, président de la Commission de la fonction publique, etc. De toute évidence, les gouvernements ont gardé une certaine confiance envers ces fonctionnaires même s'ils n'ont plus eu à collaborer directement avec un ministre.

En fait, si l'on additionne les sous-ministres maintenus en fonction et les cas de mobilité horizontale, on arrive à ce résultat : 13 sous-ministres sur 22 sont restés au service du gouvernement du Québec tout au long du premier mandat du Parti québécois, et 10 sur 23 tout au long du mandat libéral. Pour ces sous-ministres, le changement de parti au pouvoir a 
donc été marqué par un réaménagement de carrière plutôt que par un départ.

C'est encore une fois l'hypothèse de l'effet de transition qui offre la meilleure grille de lecture du tableau 2. On y voit que les rétrogradations de sous-ministres ont été plus nombreuses après la prise du pouvoir par le Parti québécois en 1976 et par le Parti libéral en 1985 qu'après la réélection du Parti québécois en 1981. Les sous-ministres en titre qui ont été rétrogradés se sont retrouvés le plus souvent sousministres adjoints ou à un poste de second ordre dans un organisme public. De même, la proportion de sous-ministres maintenus en fonction ou mutés à des postes de prestige a été plus élevée lors du second mandat péquiste (78\%) que lors du premier (59\%) ou du mandat libéral (43\%).

Quant à l'hypothèse d'un effet de différenciation partisane, elle trouve peu d'appui. On ne constate pas de différence significative en ce qui concerne le nombre de rétrogradations : cinq sous le premier mandat du Parti québécois, contre quatre sous le mandat libéral. Par contre, la proportion des sousministres maintenus à leur poste ou mutés à des postes de prestige a été plus faible sous les libéraux 110 sur 23 soit $43 \%$ ) que sous le premier mandat péquiste (13 sur 22 soit $59 \%)$. Le remaniement a donc été légèrement plus important sous les libéraux, mais la différence est mince.

En résumé, au Québec, le modèle du grand commis achoppe sur le critère de la permanence à leur poste des fonctionnaires de haut rang. Il est desservi par l'effet de transition : l'arrivée d'un nouveau gouvernement entraîne un renouvellement presque complet du personnel des sous-ministres. À l'inverse, la réélection d'un gouvernement semble stabiliser la haute fonction publique. Sur ce plan, les différences entre partis sont minimes, et c'est tout juste si l'on peut dire que le remaniement libéral a été plus important que celui opéré par le Parti québécois lors de son premier mandat. 
Tableau 2 Situation en fin de mandat des SM en poste au début du mandat

\section{Mandat}

Situation

Péquiste 1 Péquiste 2 Libéral

Encore sous-ministre

3

12

3

$(14 \%)$

(55\%)

$(13 \%)$

Rétrogradation

\begin{tabular}{|c|c|}
\hline $\begin{array}{c}5 \\
(23 \%)\end{array}$ & $\begin{array}{c}1 \\
\left(5^{\circ} \%\right)\end{array}$ \\
\hline
\end{tabular}

Mobilité horizontale

10

$(45 \%)$

5

(23\%)

7

Autre secteur public

2

(9\%)

3

(14\%)

Secteur privé

2

(9\%)

0

Retraite

Total*
1

(5\%)

0

22

(102\%)
5

\section{(30\%)}

(22\%)

2

(9\%)

2

(9\%)

$(100 \%) \quad(102 \%) \quad(100 \%)$

- Les pourcentages ayant été arrondis, leur total peut ne pas égaler $100 \%$. 


\section{L'identification politique}

Par qui les sous-ministres sortants ont-ils été remplacés? Selon le modèle du grand commis, ils auraient dû l'être par des fonctionnaires de carrière, sans passé politique. Selon le modèle de l'outsider politique, des partisans du nouveau pouvoir auraient pu leur succéder. En fait, le Parti québécois, lors de son premier mandat, n'a nommé au rang de sousministre qu'un seul de ses membres en vue. II s'agit de Louis Bernard, directeur du cabinet de René Lévesque pendant la première année de son mandat, détenteur d'un doctorat en gestion et ancien sous-ministre adjoint. Bien que Louis Bernard ait été nommé au poste le plus important, celui de secrétaire général - qui agit comme principal conseiller du Premier ministre en matière de gestion du personnel supérieur - on peut conclure que ce cas unique ne représente pas une dérogation au principe de non-partisanerie dans le recrutement des hauts fonctionnaires. Un ancien chef de cabinet libéral a même été nommé sous-ministre lors de ce premier mandat péquiste. Lors du second mandat péquiste, aucun des sousministres nommés n'avait mené par le passé une activité politique officielle.

En comparaison, le Premier ministre Robert Bourassa a nommé au rang de sous-ministre en titre six personnes ayant servi dans le cabinet d'un ministre libéral dans les années 70 (quatre au provincial et deux au fédéral). Parmi ces personnes, on remarque Benoît Morin, ancien chef de cabinet du Premier ministre Robert Bourassa de 1973 à 1976, nommé par ce dernier secrétaire général au Conseil exécutif, le 3 novembre 1986. Le phénomène semble se répéter au second mandat puisque deux autres anciens membres de cabinets libéraux ont à leur tour accédé au rang de sous-ministre en titre après le 24 septembre 1989. Bien qu'un ancien membre du cabinet de Jacques Parizeau ait été nommé sous-ministre des Finances, et même si on peut expliquer en partie ces nominations partisanes par le fait que le Parti libéral disposait en 1985 d'un bassin important d'anciens attachés politiques (ce qui n'était évidement pas le cas du Parti québécois en 
1976), on trouve ici le signe d'une certaine prise de distance des libéraux vis-à-vis du modèle du grand commis apolitique.

Cette prise de distance est toute relative cependant, car pour six nominations à caractère politique lors du mandat de 1985-1989, le Premier ministre Bourassa a procédé pendant le même temps à 27 autres nominations dont les titulaires $n$ 'ont pas eu de passé politique officiel. De plus, les six titulaires de ces nominations politiques remplissent, selon leur dossier, les exigences de la fonction, tant sur le plan de la scolarité - avec un Ph.D., trois M.A. et un diplômé du Barreau - que de l'expérience puisqu'ils avaient en moyenne quatorze années d'expérience dans la fonction publique.

Ces nuances étant faites, il reste qu'avec 6 nominations à caractère explicitement politique sur 33 nominations, le Parti libéral a élevé le taux de politisation à $18 \%$, ce qui représente près d'une nomination sur cinq. Or, nous avons vu que ce même taux s'est plutôt maintenu autour de $10 \%$ depuis le début des années 70 si l'on prend en compte l'ensemble des sous-ministres - y compris les adjoints et les associés. Étant donné que la nomination au rang de sous-ministre est, selon la tradition, moins entachée de partisanerie que des nominations inférieures, on peut en déduire que le Parti libéral, en puisant de la sorte dans son bassin d'attachés politiques, a modifié la pratique habituelle.

En résumé, si l'on se place du point de vue du modèle du grand commis, les sous-ministres québécois réussissent mieux le test de l'apolitisme formel que celui de la permanence. II semble que nos gouvernements se méfient des hauts fonctionnaires laissés en place par le parti rival et qu'ils favorisent souvent les candidats partageant leurs orientations politiques (ce que nos entrevues confirment). Mais les nominations de partisans reconnus sont tout de même rares. Toutefois, elles tendent à augmenter depuis 1985, ce qui annonce peut-être un affaiblissement continu du modèle du grand commis. Une différence entre partis apparaît ainsi pour la première fois de façon nette, le Parti libéral ayant nommé plus de partisans reconnus que ne l'a fait le Parti québécois. 


\section{La provenance des sous-ministres en titre}

Lorsqu'un gouvernement veut nommer un sous-ministre en titre à la tête d'un ministère donné, il peut soit procéder à une mutation (choisir un candidat déjà sous-ministre en titre dans un autre ministère), soit procéder à une promotion (choisir un sous-ministre adjoint ou associé), soit regarder du côté des dirigeants des réseaux (enseignement, hôpitaux, entreprises publiques) ou des autres administrations publiques ou encore regarder du côté du secteur privé. Le modèle du grand commis est identifié aux deux premières solutions, celui de l'outsider politique à la dernière, tandis que la troisième est plutôt intermédiaire entre les deux modèles.

Le tableau 3 montre les solutions choisies par nos gouvernements lors des trois mandats considérés. II indique les postes qu'occupaient les sous-ministres juste avant leur nomination. Sans conteste, le modèle du grand commis est celui qui prévaut du point de vue de la provenance immédiate. En effet, les nominations "classiques" - mutation d'un sousministre en titre, promotion d'un sous-ministre adjoint ou associé - sont de loin les plus fréquentes. Elles ont formé respectivement $71 \%, 85 \%$ et $70 \%$ des nominations effectuées au cours des trois mandats.

Le même tableau indique que trois dirigeants d'organismes autonomes ont été nommés sous-ministres en titre par le Parti québécois, six par les libéraux. Pour cinq de ces hauts fonctionnaires, l'affectation à la tête de l'administration d'un ministère a représenté un retour aux sources puisqu'ils avaient été antérieurement sous-ministres adjoints ou sous-ministres en titre. Techniquement, on peut les ajouter aux nominations classiques mentionnées au paragraphe précédent.

Fait à noter, aucun membre de cabinet n'a accédé directement au rang de sous-ministre en titre. Les libéraux ont nommé leurs six anciens membres de cabinet au poste de sous-ministre adjoint ou associé avant de les faire accéder à l'échelon supérieur.

On voit au tableau 3 que le Parti québécois, lors de son premier mandat, a promu au rang de sous-ministre en titre trois fonctionnaires qui n'avaient pas été auparavant sous- 
ministres adjoints au associés. Les bénéficiaires de ces promotions accélérées $n$ 'avaient aucun passé politique officiel.

Le tableau nous révèle aussi que le gouvernement péquiste a fait appel à cinq universitaires, alors que le gouvernement libéral n'en a recruté aucun. Le caractère intellectuel de la formation souverainiste s'est d'ailleurs traduit par une scolarité plus élevée : on y dénombre quinze sous-ministres détenteurs d'un doctorat contre deux seulement dans la formation libérale.

Par ailleurs, le tableau 3 nous fait découvrir que le réseau de la santé et des services sociaux de même que les administrations fédérales et municipales ne sont pas des voies d'accès au premier échelon administratif d'un ministère québécois. La haute fonction publique québécoise est un milieu clos qui préserve son recrutement interne, quel que soit le parti au pouvoir. Les nominations de gestionnaires issus du secteur privé sont très peu nombreuses. On n'en compte que deux durant toute la durée du gouvernement péquiste, dont un avocat de la pratique privée et un seul véritable gestionnaire, vice-président d'une grande entreprise. Le gouvernement libéral, pour sa part, a recruté au poste de sous-ministre en titre un avocat de la pratique privée et deux gestionnaires d'entreprise. Mais ces gestionnaires du privé étaient d'anciens agents de l'État, cumulant ensemble pas moins de 28 années d'expérience dans la fonction publique! Décidément, les outsiders se font rares au Québec.

Cependant, il est possible qu'un gouvernement nomme des outsiders d'abord aux seconds échelons de la hiérarchie avant de les faire accéder, après une période de formation plus ou moins brève, au rang de sous-ministre en titre. Ils sont ce qu' on peut appeler des "outsiders différés". Pour les retracer, nous avons examiné le parcours professionnel de ceux qui sont passés du rang de sous-ministre adjoint ou associé à celui de sous-ministre. Nos observations sont les suivantes:

- Premier mandat du Parti québécois. Parmi les vingt-cinq sous-ministres adjoints ou associés promus au rang de sous-ministres en titre pendant ce mandat (tableau 3), dixneuf 
Tableau 3 Dernier poste occupé avant la nomination

\section{Mandat}

Dernier poste

Péquiste 1 Péquiste 2 Libéral

Sous-ministre en titre

2

(5\%)

25
$(66 \%)$

associé

Gestionnaire (organismes autonomes)

$\begin{array}{ccc}1 & 2 & 6 \\ (3 \%) & \left(8^{2} \%\right) & (18 \%)\end{array}$

0

0

valent dans un ministère $(8 \%)$

Enseignant, administrateur 4 scolaire ou universitaire (11\%)

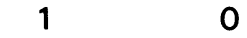

(4\%)

Gestionnaire (santé, services sociaux)

$(3 \%)$

0

1

(3\%)

Gestionnaire (autres admi- 0 nistrations publiques)

(4 1

0

Gestionnaire (secteur privé) 2

$(5 \%)$

0

3

(9\%)

Total"

38

(101\%)

26

33

$(101 \%) \quad(100 \%)$

\footnotetext{
- Les pourcentages ayant été arrondis, leur total peut ne pas égaler $100 \%$.
} 
étaient déjà adjoints ou associés lors de la prise du pouvoir par le Parti québécois, quatre autres ont progressé normalement dans leur carrière avant d'accéder aux échelons supérieurs de la hiérarchie sous le gouvernement péquiste. Tel est le cas de Louis Bernard, dont nous avons déjà parlé : haut fonctionnaire au début des années 70 , il entre au service de l'opposition péquiste, devient chef de cabinet du Premier ministre Lévesque avant de regagner la haute fonction publique comme secrétaire général associé, puis comme secrétaire général. II ne reste plus qu'un seul cas d'outsider différé, encore un universitaire, qui fut nommé directement sous-ministre adjoint avant d'être promu sous-ministre en titre un an plus tard. On ne lui connaît pas de passé politique officiel.

- Second mandat du Parti québécois. Deux cas seulement retiennent I'attention. II s'agit encore d'universitaires nommés sous-ministres en titre après avoir été quelques années sous-ministres associés. Leur curriculum n'indique aucune activité politique officielle.

- Mandat libéral. On retrouve deux fonctionnaires de carrière qui ont rejoint le secteur privé durant les années péquistes, puis sont revenus à la fonction publique comme sous-ministres adjoints après le retour des libéraux (I'un d'eux a un passé libéral officiel). Un autre fonctionnaire de carrière provient d'une autre province et avait, dans les années 70, servi dans le cabinet du Premier ministre Trudeau. Enfin, on relève un cas intéressant, celui d'un directeur de cabinet d'un ministre liberral de 1974 à 1976. Gestionnaire dans une firme privée durant sept ans, puis sous-ministre adjoint pendant six mois en 1986, il est devenu ensuite sous-ministre en titre. C'est le seul exemple d'outsider politique véritable que l'on a relevé au cours des trois mandats. Le seul qui réunisse les critères de provenance extérieure et d'identification partisane.

Si l'on dresse le bilan de la provenance des sous-ministres nommés lors des trois mandats, on n'en trouve que deux lors du premier mandat péquiste et un lors du mandat libéral qui soient venus du secteur privé et aient atterri directement à la 
tête d'un ministère. Si on leur ajoute les huit universitaires et le gestionnaire du secteur de la santé devenus sous-ministres en titre (sous le Parti québécois) directement ou après un court stage comme adjoints, ainsi que le fonctionnaire d'une autre province et le gestionnaire du secteur privé placés sousministres adjoints, puis sous-ministres par les libéraux, cela ne fait que quatorze sous-ministres sur $84(17 \%)$ qui n'étaient pas fonctionnaires de carrière dans le gouvernement du Québec. Un taux de recrutement interne de $83 \%$, voilà un trait marquant de la haute fonction publique québécoise, qui l' «arrime" au modèle du grand commis.

À côté de cette forte constante les différences entre mandats ou entre partis paraissent ténues. À mettre au compte de l'effet de transition, il y a le fait que c'est lors du second mandat du Parti québécois que la proportion de nominations classiques (mutations de sous-ministres en titre ou promotion d'adjoints ou d'associés) a été la plus élevée. Le Parti québécois a travaillé avec un personnel de hauts fonctionnaires qu'il avait largement façonné au cours de son premier mandat. Comme signe d'une différence entre partis, on remarque que le Parti québécois, surtout à ses débuts, a puisé dans le bassin universitaire, tandis que le Parti libéral a rapatrié dans les ministères une demi-douzaine de dirigeants d'organismes autonomes. On trouve aussi une certaine différence sur le plan de la formation des sous-ministres : $47 \%$ de ceux nommés par les libéraux détenaient un diplôme en administration ou en science économique, comparativement à $34 \%$ et $32 \%$ lors des deux mandats péquistes. Le style de chaque parti apparaît ainsi en filigrane, mais le trait fort demeure immuable : la haute fonction publique est le giron du gouvernement, quel que soit le parti au pouvoir.

\section{Conclusion}

Les ministres passent, les hauts fonctionnaires restent, diton. Cet adage ne correspond pas à la réalité québécoise. Un changement de gouvernement provoque un renouvellement presque complet des chefs administratifs des ministères. Une poignée demeure en fonction, plusieurs sont replacés à des postes intéressants dans les organismes publics, mais beaucoup partent ou sont rétrogradés. Ce constat est d'autant 
plus important qu'il touche le plus haut fonctionnaire de la hiérarchie, soit le sous-ministre en titre, celui-là même à qui la tradition délègue la continuité de l'État.

Le Premier ministre québécois remplace les hauts fonctionnaires parce qu'il recherche des collaborateurs qui partagent ses orientations ou ses programmes, qui ne sont pas compromis avec les politiques du gouvernement précédent, qui correspondent davantage à son style de gestion ou, encore, qui puissent travailler dans I'harmonie avec ses ministres. $D^{\prime}$ où, sans doute, le taux de roulement élevé après un changement de gouvernement. Mais rien $n$ 'indique que cette préoccupation politique se fasse au détriment de la compétence : les rares sous-ministres qui ont eu un passé politique officiel ont les compétences et l'expérience requises pour administrer un ministère. Responsable du ministère, principal conseiller du ministre, le sous-ministre est un personnage trop utile pour qu'il en soit autrement.

S'il y a politisation de la haute fonction publique québécoise, celle-ci est de nature "fonctionnelle» plutôt que "partisane». Elle vise à l'action concertée de l'équipe politico-administrative au sommet du gouvernement, et non à l'élargissement du réservoir d'emplois des amis du régime. Elle implique une certaine distance vis-à-vis de l'apolitisme strict du grand commis, mais elle demeure très éloignée du modèle de fonctionnaire politique que l'on retrouve dans d'autres traditions administratives. Toutefois, la nomination d'une demi-douzaine d'attachés politiques, au retour des libéraux en 1985, constitue un jalon en ce sens qui ferait, s'il devait s'élargir, une espèce menacée du grand commis québécois.

Sauf quelques rares exceptions, on ne devient pas sousministre sans avoir l'expérience du secteur public. Très peu de sous-ministres ont eu une expérience prolongée de gestionnaires dans le secteur privé. La vie professionnelle de la majorité d'entre eux s'est déroulée pour l'essentiel au sein du gouvernement. C'est là une caractéristique marquée de notre système administratif, dont il faudrait étudier les avantages (continuité?), les inconvénients (fermeture sociale?) et les causes lécart salarial avec le secteur privé? isolement de la Capitale? pouvoir du fonctionnariat?).

Les sous-ministres québécois sont tout de même des grands commis fragiles. Formellement apolitiques, carriéris- 
tes, ils survivent mal à l'alternance des gouvernements. D' où certains paradoxes. Les gouvernements les renouvellent, et pourtant leurs remplaçants semblent être leurs pareils, issus de la même filière bureaucratique. La très haute fonction publique est mobile, presque éphémère, et pourtant elle gagne de plus en plus en expérience : les sous-ministres nommés lors du premier mandat péquiste avaient derrière eux, au moment de leur nomination, en moyenne 8,6 années dans la fonction publique, comparativement a 11,7 pour leurs homologues du second mandat et a 13,9 pour ceux du mandat libéral. Elle vieillit aussi, son âge moyen passant de 41 à 46, puis a 47 ans au fil des mandats.

En somme, les gouvernements remplacent une proportion importante de très hauts fonctionnaires quand ils arrivent au pouvoir. L'hypothèse d'un effet de transition est largement confirmée. Mais les traits de la haute fonction publique, eux, ne changent qu'à la marge, au gré des partis au pouvoir : apparition de quelques universitaires sous le Parti québécois et d'une demi-douzaine d'anciens membres de cabinet sous le Parti libéral. La voie royale pour accéder aux plus hauts paliers de l'administration demeure la fonction publique, quelle que soit la couleur du gouvernement. Plus encore, cette voie royale traverse le centre névralgique de l'appareil politicoadministratif, soit les secrétariats du Conseil du trésor et du Conseil exécutif : au fil des mandats, $26 \%, 56 \%$ et $48 \%$ des sous-ministres sont passés par ces organismes centraux à un stade antérieur de leur carrière. Les hauts fonctionnaires passent, mais le modèle du grand commis, dans ses principes fondamentaux, demeure. 


\section{Bibliographie}

BERNARD, Louis, Réflexions sur l'art de se gouverner. Essai d'un praticien, Montréal, Québec-Amérique, 1987.

BOREHAM, Paul, Michael CASS et Michael McCALLUM, "The Australian Bureaucratic Elite; The Importance of Social Backgrounds and Occupational Experience», The Australian and New Zealand Journal of Sociology, vol. 15, $n^{\circ} 2,1979$, pp. 45-55.

BOURGAULT, Jacques, Répertoire des employés supérieurs (hors cadredes ministères du gouvernement du Québec 1867-1983, Québec, Bibliothèque de l'Assemblée nationale du Québec, Bibliographie et documentation, $n^{\circ}$ 16, 1984.

BOURGAULT, Jacques, Les administrateurs d'État du gouvernement du Québec 1867-1985, thèse de doctorat d'État, Paris (soutenue à la Fondation nationale des sciences politiques), 1986.

BOURGAULT, Jacques et Stéphane DION, "Les gouvernements antibureaucratiques face à la haute administration : une comparaison Québec-Canada», Politiques et Management public, vol. $7, \mathrm{n}^{\circ} 2,1989$, pp. 97-118.

BOURGAULT, Jacques et Stéphane DION, L'évolution du profil des sous-ministres fédéraux 1867-1988, rapport présenté au Centre canadien de gestion, Ottawa, Centre canadien de gestion, 1990.

DERLIEN, Hans-Ulrich, «Repercussions of Government Change on the Career Civil Service in West Germany : The Cases of 1969 and 1982», Governance, vol. 1, $n^{\circ} 1,1988$, pp. 50-78.

DIERICKX, Guido, «Bureaucrats and Politicians in Belgium : An Uneasy Relationship", Communication présentée à la réunion annuelle de l'American Political Science Association, San Francisco, 1990. 
DION, Stéphane, "La politisation des administrations publiques : éléments d'analyse stratégique», Administration publique du Canada, vol. 29, n 1, 1986, pp. 95-117.

ELDERWELD, Samuel, et al., "Elite Perceptions of the Political Process in the Netherlands. Looked at in Comparative Perspective", dans DOGAN, Mattei, éd., The Mandarins of Western Europe : The Political Role of Top Civil Servants, New York, Sage, 1975, pp. 129-161.

GOURNAY, Bernard, Introduction a la science administrative, Paris, Presses de la Fondation nationale des sciences politiques, 1978.

HECLO, Hugh, A Government of Strangers, Washington D.C., Brookings Institution, 1977.

LESAGE, Gilles, "Des mandarins à contrat», Le Devoir, 17 mai 1986.

LINDE, Claes et Peter EHN, "The Swedish Administrative Eli-te», Communication présentée à la réunion annuelle de l'American Political Science Association, San Francisco, 1990.

LEGREID, Per et Johan P. OLSEN, "Top Civil Servants in Norway", dans SULEIMAN, Ezra N., éd., Bureaucrats and Policy Making. A Comparative Overview, New York et Londres, Holmes et Meier, 1984, pp. 206-241.

PARTI LIBÉRAL DU QUÉBEC, Maîtriser l'avenir. Programme politique, 1985.

RAPPORT GOBEIL, Groupe de travail sur la révision des fonctions et des organisations gouvernementales, Québec, Gouvernement du Québec, 1986.

THEAKSTON, Kevin et Geoffrey K. FRY, «Britain's Administrative Elite : Permanent Secretaries 1900-1986», Public Administration, vol. 67, $\mathrm{n}^{\circ} 2,1989, \mathrm{pp} .129-147$.

URIO, P. et al., Sociologie politique de la haute administration publique en Suisse, Paris, Economica, 1989. 